\title{
SerialEM: A Program for Automated Tilt Series Acquisition on Tecnai Microscopes Using Prediction of Specimen Position
}

\author{
David N. Mastronarde
}

Boulder Laboratory for Three Dimensional Electron Microscopy of Cells, Dept. of Molecular, Cellular, and Developmental Biology, University of Colorado, Boulder, CO 80309

The traditional approach to automated tilt series acquisition involves tracking specimen position and measuring focus after every tilt, sometimes with time-consuming magnification changes [1]. The predictable performance of modern goniometers has allowed the development of a different method in which the translational specimen holder movements are precalibrated with a rapid, coarse tilt series, then the full tilt series is acquired relying on this calibration [2]. Although this method is efficient, with thick specimens imaged at $300 \mathrm{KV}$ in our laboratory we have found it susceptible to drift, the less accurate adjustment of eucentric height for cryo-samples, and other non-ideal features of the specimen or microscope. I thus developed a program, SerialEM, that uses a new approach based on prediction of specimen position during the tilt series from the position at previous tilts. This method achieves both the speed of pre-calibration and the reliability of the traditional approach.

The new prediction method is illustrated in Fig 1. At any given tilt angle, the program has information about the position in $\mathrm{X}, \mathrm{Y}$, and $\mathrm{Z}$ at previous tilt angles. A function is fit to each of these coordinates independently and the position on the current tilt is predicted (Fig. 1, open circles). When there are only two data points and thus no indication of the quality of the prediction (Tilt 3), the prediction is used but the position is measured and corrected with a tracking image. With more data, the program examines both the standard error of the new prediction (error bars), and the actual error of the prediction on the previous tilt (vertical distance between open and filled circles). If either of these errors is too high (Tilt 4), the program will take a tracking image; but if both errors are small enough (Tilt 5), the prediction is deemed reliable and no tracking image is taken. The final recorded image is used to measure and corrects position, to keep errors from accumulating. A similar procedure is used for focusing, except here it is necessary to measure focus periodically.

Figure 2 shows results from a typical tilt series of a $300 \mathrm{~nm}$ thick plastic section, with a tilt increment of $1^{\circ}$. The program was able to skip tracking after 3 tilts, and focus only every $5^{\circ}$ after 7 tilts. At $-35^{\circ}$ degrees, the program reset the image shift to zero and moved the stage to compensate. (An alignment flaw limits the image shift on the $300 \mathrm{KV}$ Tecnai to less than $2 \mu \mathrm{m}$ with small objective apertures.) Fig. 2 shows that after the image shift reset, tracking and focusing were needed on every tilt for the next 6 tilts, but thereafter tracking was unneeded and focusing was minimized. This tilt series took 45 minutes, of which at least 30 were required simply to record the final $2 \mathrm{~K} \mathrm{x}$ 2K images from the Gatan Megascan 795 camera. The total time is thus comparable to that required for precalibration, as is the number of pictures needed for focusing. In more difficult situations where precalibration would fail, such as with extreme drift, the series may take $10-20 \%$ longer as it tracks and focuses on a larger fraction of tilts, but it still acquires an adequately aligned tilt series. The prediction method thus allows reliable tilt series acquisition while minimizing collection time.

SerialEM runs on FEI Tecnai microscopes with Gatan cameras. It presents an integrated user interface for image acquisition, examination and storage. Many options for partially or fully 
automated data collection make the program highly flexible; e.g., the user can stop the tilt series if necessary, control the actions taken when it resumes, and even conveniently back up to a previous tilt angle and replace the stored data from that point onward. Tilt series can be acquired in low-dose mode, with focusing and tracking images taken outside the main recording area. SerialEM can also automatically montage from multiple overlapping frames, making it useful for cases where the area of interest is larger than the field of the camera. Finally, it incorporates control of a Gatan Imaging Filter (GIF) and can acquire tilt series of energy filtered images.

\section{References}

[1] A. J. Koster et al., Ultramicroscopy 46 (1992) 207-221.

[2] U. Ziese et al., J. Microscopy 205 (1991) 187-200.

[3] This work was supported by NIH/NCRR grant RR00592 and NIH Program Project grant P01GM61306 to J. Richard McIntosh.
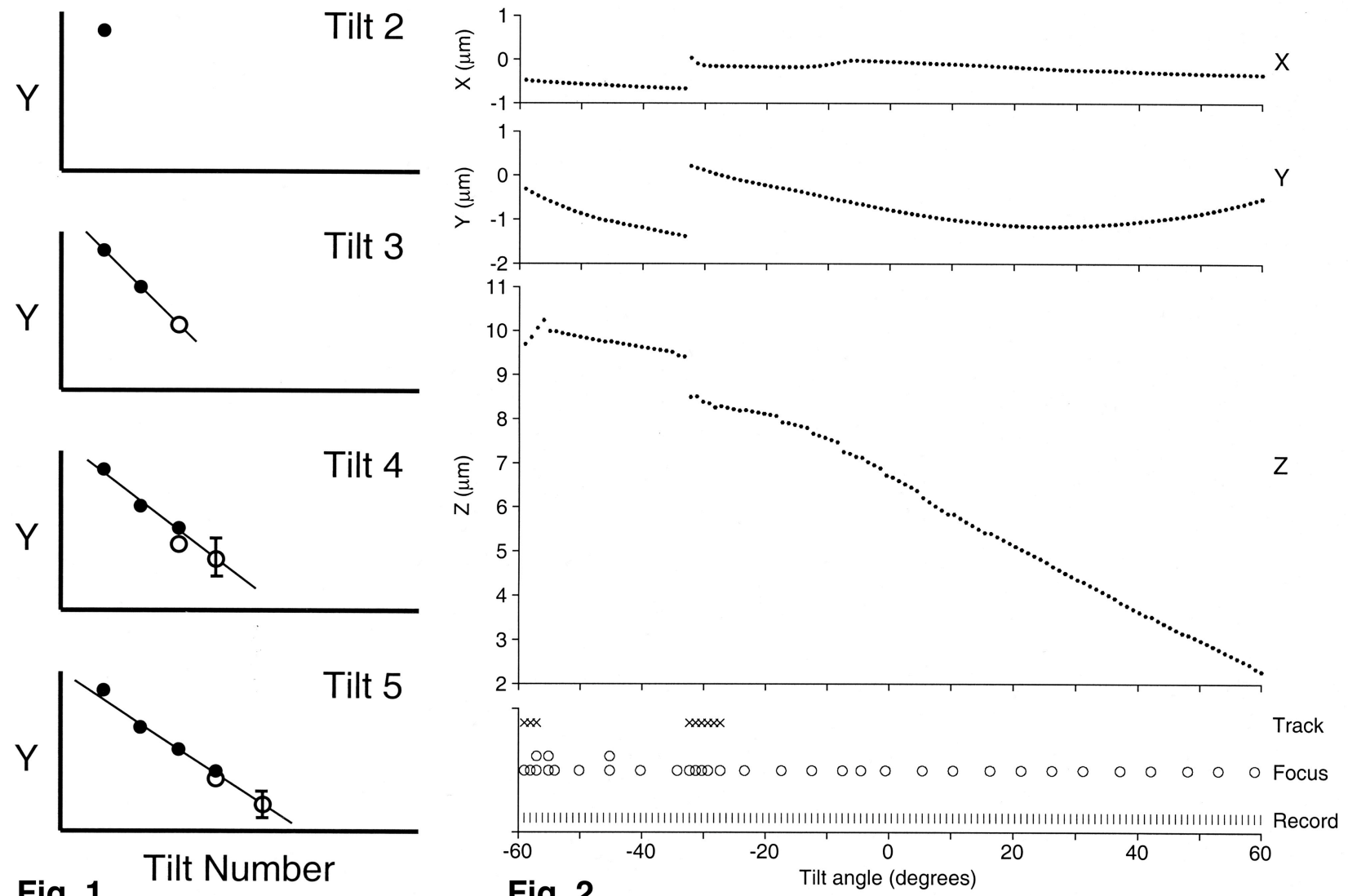

Fig. 1

Tilt Number

Figure 1. The prediction method. Y coordinate (perpendicular to the tilt axis) is plotted versus tilt number for successive tilts. Closed circles are actual measurements; open circles are predictions from previous positions.

Figure 2. The top three panels show changes in $\mathrm{X}$ and $\mathrm{Y}$ coordinates and in focus setting $(\mathrm{Z})$ during a tilt series. The lower panel shows a log of the exposures taken during the tilt series: final unbinned images (vertical ticks), tracking images binned by 4 (crosses), and focus measurements (circles). The latter involve taking three pictures to measure beam-tilt dependent image shift. 